\title{
String theories on warped AdS backgrounds and integrable deformations of spin chains
}

\author{
Takashi Kameyama, Kentaroh Yoshida \\ Department of Physics, Kyoto University, Kyoto 606-8502, Japan \\ E-mail: kame@gauge.scphys.kyoto-u.ac.jp, \\ kyoshida@gauge.scphys.kyoto-u.ac.jp
}

\begin{abstract}
We study integrable deformations of AdS/CFT by focusing upon three kinds of warped $\mathrm{AdS}_{3}$ geometries, 1) space-like warped $\mathrm{AdS}_{3}$, 2) time-like warped $\mathrm{AdS}_{3}$ and 3) null warped $\mathrm{AdS}_{3}$. These geometries are embedded into type IIB supergravity solutions and are regarded as consistent string backgrounds. By restricting the classical motion of strings on the warped $\mathrm{AdS}_{3} \times \mathrm{S}^{1}$ subspace, the Landau-Lifshitz sigma models are derived by taking the fast-moving limit. The first two warped $\mathrm{AdS}_{3}$ spaces correspond to anisotropic deformations of the $\operatorname{sl}(2)$ spin chain and the last one to Jordanian deformations. After taking the continuum limit of the deformed spin chains with coherent states, the resulting theories agree with the Landau-Lifshitz sigma models obtained from the string-theory side.
\end{abstract}

Keywords: AdS-CFT Correspondence, Sigma Models, Integrable Field Theories

ArXiv EPRINT: 1304.1286 


\section{Contents}

1 Introduction 1

2 Setup 2

2.1 String action 2

2.2 Warped $\mathrm{AdS}_{3}$ geometries 3

3 Space-like warped $\mathrm{AdS}_{3}$ and deformed spin chains 4

3.1 Fast-moving string limit 4

$\begin{array}{lll}3.2 & \text { From anisotropically deformed } \operatorname{sl}(2) \text { spin chains } & 6\end{array}$

4 Time-like warped $\mathbf{A d S}_{3}$ and deformed spin chains $\quad 7$

$\begin{array}{lll}4.1 & \text { Fast-moving string limit } & 7\end{array}$

$\begin{array}{lll}4.2 & \text { From anisotropically deformed } \operatorname{sl}(2) \text { spin chains } & 8\end{array}$

5 Null warped $\mathrm{AdS}_{3}$ and Jordanian deformations $\quad 9$

5.1 Fast-moving string limit $\quad 9$

5.2 From Jordanian deformations of the $\operatorname{sl}(2)$ spin chain $\quad 11$

6 Conclusion and Discussion $\quad 12$

$\begin{array}{ll}\text { A Convention of } \operatorname{sl}(2) \text { generators } & 13\end{array}$

$\begin{array}{ll}\text { B The } \operatorname{sl}(2) \text { coherent states } & 14\end{array}$

$\begin{array}{ll}\text { C The continuum limit of the } \operatorname{sl}(2) \text { spin chain } & 14\end{array}$

\section{Introduction}

The AdS/CFT correspondence [1-3] is still the focus of attention though it has been studied exhaustively for more than the decade. It is now supported by an enormous amount of evidence. The discovery of the integrable structure behind AdS/CFT is a truly tremendous achievement toward the complete proof. The results on this issue have been accumulated in the great review [4].

The next step is to consider integrable deformations of AdS/CFT. The usual AdS/CFT belongs to the rational class of integrable systems. For example, the XXZ models, which are anisotropic deformations of the XXX model, shed light on the deeper integrable structure like quantum affine algebras, while the XXX model is nothing but a degenerate limit of the XXZ models. One may expect that a similar thing would happen in the case of AdS/CFT and hence it is of importance to consider integrable deformations of AdS/CFT. 
In the recent, some works have been done in this direction. There are mainly two approaches. The one is based on $q$-deformations of S-matrix of the integrable spin chain [5-9]. The other is to consider deformations of target spaces of string sigma models, namely, warped AdS spaces and/or deformed spheres. For earlier works and recent progress along this direction, for example, see [10-12] and [13-20], respectively.

We follow here the latter approach and study integrable deformations of AdS/CFT by focusing upon three kinds of warped $\mathrm{AdS}_{3}$ geometries, 1) space-like warped $\mathrm{AdS}_{3}$ [21], 2) time-like warped $\mathrm{AdS}_{3}[22,23]$ and 3) null warped $\mathrm{AdS}_{3}$ [24]. The third one is also known as three-dimensional Schrödinger spacetimes $[25,26]$. For a summary of warped $\mathrm{AdS}_{3}$, see [27]. All of these geometries are embedded into supergravity solutions and are regarded as consistent string backgrounds ${ }^{1}$. By restricting the classical motion of strings on the warped $\mathrm{AdS}_{3} \times \mathrm{S}^{1}$ subspace, the Landau-Lifshitz sigma models are derived by taking the fast-moving string limit $[35,36]$.

The first two warped $\mathrm{AdS}_{3}$ geometries correspond to anisotropic deformations of the $s l(2)$ spin chain. The last one corresponds to Jordanian deformations, which mean null-like deformations. After taking the continuum limit of the deformed spin chains with coherent states, the resulting theories agree with the Landau-Lifshitz sigma models obtained from the string-theory side.

This note is organized as follows. Section 2 is the setup of the string action and overviews three kinds of warped $\mathrm{AdS}_{3}$. In section 3 we argue the correspondence between strings on space-like warped $\mathrm{AdS}_{3} \times \mathrm{S}^{1}$ and anisotropic deformations of the $s l(2)$ spin chain. By taking a fast moving limit of the relativistic string action, the Landau-Lifshitz sigma model action is derived. Then the continuum limit of the deformed spin chains is taken with coherent states. The both results show the agreement. In section 4 we consider the time-like warped $\mathrm{AdS}_{3}$ case. The analysis is almost the same as in section 3. In section 5 we consider the null warped $\mathrm{AdS}_{3}$ case, where one has to consider Jordanian deformations of the $\operatorname{sl}(2)$ spin chain. The agreement of the Landau-Lifshitz sigma models is shown again. Section 6 is devoted to conclusion and discussion. The convention of $\operatorname{sl}(2)$ generators is summarized in Appendix A. The basic property of $\operatorname{sl}(2)$ coherent states is introduced in Appendix B. The continuum limit of the undeformed part of the $\operatorname{sl}(2)$ spin chain is reviewed in Appendix C.

\section{Setup}

We first introduce the string action and fix our notation. Then three kinds of warped $\mathrm{AdS}_{3}$ are summarized to make our explanation clear.

\subsection{String action}

The string action we consider here is given by

$$
S=-\frac{1}{4 \pi \alpha^{\prime}} \int d \tau d \sigma \eta^{\mu \nu}\left[\partial_{\mu} X^{M} \partial_{\nu} X^{N} G_{M N}\right]
$$

\footnotetext{
${ }^{1}$ For space-like and time-like warped $\mathrm{AdS}_{3}$, see [21, 28-31]. For the null case, see [32-34].
} 
where the string world-sheet coordinates are $\sigma^{\mu}=(\tau, \sigma)$ with $\eta_{\mu \nu}=(-1,+1)$. The target space metric is given by $G_{M N}$ and $X^{M}(\tau, \sigma)$ are the embedding coordinates of the string world-sheet into the target space. The periodic boundary condition is imposed for the spatial direction of the string world-sheet,

$$
X^{M}(\tau, \sigma+2 \pi)=X^{M}(\tau, \sigma) .
$$

It is necessary to take account of the Virasoro constraints,

$$
\begin{gathered}
G_{M N} \partial_{\tau} X^{M} \partial_{\sigma} X^{N}=0 \\
G_{M N}\left(\partial_{\tau} X^{M} \partial_{\tau} X^{N}+\partial_{\sigma} X^{M} \partial_{\sigma} X^{N}\right)=0,
\end{gathered}
$$

which will be important in reducing the system to the Landau-Lifshitz sigma models.

\subsection{Warped $\mathrm{AdS}_{3}$ geometries}

There are three kinds of warped $\mathrm{AdS}_{3}$ geometries, 1) space-like warped $\mathrm{AdS}_{3}, 2$ ) time-like warped $\mathrm{AdS}_{3}$ and 3) null warped $\mathrm{AdS}_{3}$. The metrics of them are listed below.

\section{1) space-like warped $\mathrm{AdS}_{3}$}

The metric of space-like warped $\mathrm{AdS}_{3}$ is given by

$$
d s^{2}=\frac{R^{2}}{4}\left[-\cosh ^{2} \rho d t^{2}+d \rho^{2}+(1-C)(d u+\sinh \rho d t)^{2}+d \varphi^{2}\right],
$$

where $C$ is a constant, deformation parameter. The three variables $t, \rho, u$ describe the usual $\mathrm{AdS}_{3}$ with the radius $R$ in the global coordinates when $C=0$.

The metric (2.3) can be rewritten as

$$
d s^{2}=\frac{R^{2}}{2}\left[\operatorname{Tr}(J J)-2 C \operatorname{Tr}\left(T^{1} J\right) \operatorname{Tr}\left(T^{1} J\right)\right],
$$

in terms of the left-invariant one-form $J=g^{-1} d g$ with the group element

$$
g=\mathrm{e}^{-t T^{0}} \mathrm{e}^{\rho T^{2}} \mathrm{e}^{u T^{1}} .
$$

For the definition of $T^{a}$ 's, see Appendix A. Note that the expression (2.4) gives rise to the definition of space-like warped $\mathrm{AdS}_{3}$, independently of the coordinates.

\section{2) time-like warped $\mathrm{AdS}_{3}$}

The metric of time-like warped $\mathrm{AdS}_{3}$ is given by

$$
d s^{2}=\frac{R^{2}}{4}\left[\cosh ^{2} \rho d u^{2}+d \rho^{2}-(1+C)(d t+\sinh \rho d u)^{2}+d \varphi^{2}\right] .
$$

With the group element

$$
g=\mathrm{e}^{u T^{1}} \mathrm{e}^{\rho T^{2}} \mathrm{e}^{-t T^{0}}
$$

the metric (2.6) can be rewritten as

$$
d s^{2}=\frac{R^{2}}{2}\left[\operatorname{Tr}(J J)-2 C \operatorname{Tr}\left(T^{0} J\right) \operatorname{Tr}\left(T^{0} J\right)\right] .
$$




\section{3) null warped $\mathrm{AdS}_{3}$}

The metric of null warped $\mathrm{AdS}_{3}$ is given by

$$
d s^{2}=\frac{R^{2}}{4}\left[-2 \mathrm{e}^{-\rho} d U d V+d \rho^{2}-C \mathrm{e}^{-2 \rho} d V^{2}\right] .
$$

The metric (2.9) is written as deformations with the $T^{-}$component,

$$
d s^{2}=\frac{R^{2}}{2}\left[\operatorname{Tr}(J J)-2 C \operatorname{Tr}\left(T^{-} J\right) \operatorname{Tr}\left(T^{-} J\right)\right] .
$$

When the group element $g$ is parametrized as

$$
g=\mathrm{e}^{V T^{+}} \mathrm{e}^{\rho T^{2}} \mathrm{e}^{U T^{-}},
$$

the metric (2.9) is reproduced.

\section{Space-like warped $\mathrm{AdS}_{3}$ and deformed spin chains}

\subsection{Fast-moving string limit}

We consider the fast-moving limit of strings propagating on space-like warped $\mathrm{AdS}_{3} \times \mathrm{S}^{1}$. We first have to change the coordinates of the metric (2.3). The reason is that the warped $\mathrm{AdS}_{3}$ is expressed as a $U(1)$ fibration over $\mathrm{AdS}_{2}$ and it is not convenient to take the limit.

Let us take the following parametrization of $g$, instead of (2.5),

$$
g=\mathrm{e}^{-(\phi+t) T^{0}} \mathrm{e}^{2 \rho T^{1}} \mathrm{e}^{(\phi-t) T^{0}} .
$$

Then, with an $\mathrm{S}^{1}$ circle described by $\varphi$, the metric of the warped $\mathrm{AdS}_{3} \times \mathrm{S}^{1}$ is given by

$$
\begin{aligned}
d s^{2}=R^{2} & {\left[-\cosh ^{2} \rho d t^{2}+d \rho^{2}+\sinh ^{2} \rho d \phi^{2}+d \varphi^{2}\right.} \\
& \left.-\frac{C}{4}[2 \cos (\phi-t) d \rho-\sin (\phi-t) \sinh 2 \rho(d \phi+d t)]^{2}\right] .
\end{aligned}
$$

Note that the deformation part looks more complicated than the metric (2.3), but the metric (3.2) is convenient to take the fast-moving limit, as we will see below.

Next we perform the coordinate transformation

$$
\phi=\tilde{\phi}+t, \quad \varphi=\tilde{\varphi}+t, \quad \rho=\frac{1}{2} \tilde{\rho},
$$

and take the gauge $t=\kappa \tau$. Then we take the following limit,

$$
\kappa \rightarrow \infty \quad \text { with } \quad \kappa^{2} C, \quad \kappa \partial_{\tau} \tilde{\phi}, \quad \kappa \partial_{\tau} \tilde{\varphi}, \quad \kappa \partial_{\tau} \tilde{\rho}: \text { fixed } .
$$

Note that the above limit (3.4) contains an additional condition on $\kappa^{2} C$ in comparison to the usual one [35-37]. This condition is necessary to ensure the finiteness of the resulting action as mentioned in [13]. 
After all, the resulting action of the warped $\mathrm{AdS}_{3} \times \mathrm{S}^{1}$ is given by

$$
\begin{array}{r}
S=\frac{R^{2}}{4 \pi \alpha^{\prime}} \int d \tau d \sigma[- \\
-\kappa^{2} C \sin ^{2} \tilde{\phi} \sinh ^{2} \tilde{\rho}+\kappa\left[(\cosh \tilde{\rho}-1) \partial_{\tau} \tilde{\phi}+2 \partial_{\tau} \tilde{\varphi}\right] \\
\left.-\frac{1}{4}\left[\left(\partial_{\sigma} \tilde{\rho}\right)^{2}+2(\cosh \tilde{\rho}-1)\left(\partial_{\sigma} \tilde{\phi}\right)^{2}\right]-\left(\partial_{\sigma} \tilde{\varphi}\right)^{2}\right] .
\end{array}
$$

The Virasoro constraints are also changed under the limit (3.4). To the leading order in $\kappa$, the first Virasoro constraint (2.1) becomes

$$
0=\kappa\left[(\cosh \tilde{\rho}-1) \partial_{\sigma} \tilde{\phi}+2 \partial_{\sigma} \tilde{\varphi}\right] .
$$

By eliminating $\partial_{\sigma} \tilde{\varphi}$ from (3.5) with (3.6), the leading-order action is given by

$$
\begin{gathered}
S=\frac{R^{2}}{4 \pi \alpha^{\prime}} \int d \tau d \sigma\left[-\kappa^{2} C \sin ^{2} \tilde{\phi} \sinh ^{2} \tilde{\rho}+\kappa\left[(\cosh \tilde{\rho}-1) \partial_{\tau} \tilde{\phi}+2 \partial_{\tau} \tilde{\varphi}\right]\right. \\
\left.-\frac{1}{4}\left[\left(\partial_{\sigma} \tilde{\rho}\right)^{2}+\sinh ^{2} \tilde{\rho}\left(\partial_{\sigma} \tilde{\phi}\right)^{2}\right]\right] .
\end{gathered}
$$

Note that the action (3.7) is written in the non-relativistic form in the sense of the string world-sheet. That is, it contains only the first order derivative on the world-sheet time $\tau$.

For later purpose, it is helpful to rewrite the action (3.7) by introducing new parameters

$$
L \equiv \frac{R^{2} \kappa}{2 \pi \alpha^{\prime}}, \quad \lambda \equiv \frac{R^{4}}{\alpha^{\prime 2}} .
$$

Then the action is rewritten $\operatorname{as}^{2}$

$$
\begin{array}{r}
S=\frac{L}{2} \int d t d \sigma\left[-C \sin ^{2} \tilde{\phi} \sinh ^{2} \tilde{\rho}+\left[(\cosh \tilde{\rho}-1) \partial_{t} \tilde{\phi}+2 \partial_{t} \tilde{\varphi}\right]\right. \\
\left.-\frac{\lambda}{16 \pi^{2} L^{2}}\left[\left(\partial_{\sigma} \tilde{\rho}\right)^{2}+\sinh ^{2} \tilde{\rho}\left(\partial_{\sigma} \tilde{\phi}\right)^{2}\right]\right]
\end{array}
$$

where the time variable has been changed from $\tau$ to $t$ through $t=\kappa \tau$. As a result, a new potential term has been added. When $C=0$, the result in $[36,37]$ is reproduced.

Finally let us see the equations of motion,

$$
\begin{array}{r}
C \sin 2 \tilde{\phi} \sinh ^{2} \tilde{\rho}+\sinh \tilde{\rho} \partial_{t} \tilde{\rho}-\frac{\lambda}{8 \pi^{2} L^{2}} \partial_{\sigma}\left(\partial_{\sigma} \tilde{\phi} \sinh ^{2} \tilde{\rho}\right)=0, \\
-C \sin ^{2} \tilde{\phi} \sinh \tilde{2 \rho}+\sinh \tilde{\rho} \partial_{t} \tilde{\phi}+\frac{\lambda}{8 \pi^{2} L^{2}}\left[\partial_{\sigma}^{2} \tilde{\rho}-\frac{1}{2} \sinh 2 \tilde{\rho}\left(\partial_{\sigma} \tilde{\phi}\right)^{2}\right]=0 .
\end{array}
$$

These are identical to the Landau-Lifshitz equations ${ }^{3}$

$$
-\partial_{t} \vec{n}=\frac{\lambda}{8 \pi^{2} L^{2}} \vec{n} \times \partial_{\sigma}^{2} \vec{n}+\vec{n} \times \mathcal{J} \vec{n}
$$

\footnotetext{
${ }^{2}$ The similar action is derived also in [13]. However, the deformation term we obtained is different from the one in [13] by $\sin ^{2} \tilde{\phi}$. This factor is necessary to see the agreement with the result from the continuum limit of the deformed $s l(2)$ spin chains.

${ }^{3}$ Note that $(\vec{n} \times \vec{m})_{i}=\varepsilon_{i j k} n^{j} m^{k}$. Here we have introduced the totally antisymmetric tensor $\varepsilon_{i j k}$ with $\varepsilon_{012}=+1$ and the vector $\vec{n}$ parameterizes $\operatorname{sl}(2)$ coherent states. The indices of the vector are raised and lowered with $\eta^{i j}=\operatorname{diag}(+1,-1,-1)$. For the detail of $s l(2)$ coherent states, see Appendix B.
} 
with the anisotropic matrix $\mathcal{J}$

$$
\mathcal{J}^{i j}=\operatorname{diag}(j,-(j-2 C),-j) \quad(j: \text { an arbitrary const. }) .
$$

Note that the new potential term in (3.9) is related to the following quantity,

$$
\mathcal{J}^{i j} n_{i} n_{j}=j+2 C \sin ^{2} \tilde{\phi} \sinh ^{2} \tilde{\rho} .
$$

\subsection{From anisotropically deformed $\operatorname{sl}(2)$ spin chains}

It is a turn to examine the continuum limit of the deformed $\operatorname{sl}(2)$ spin chains with $\operatorname{sl}(2)$ coherent states.

The Hamiltonian of anisotropically deformed $s l(2)$ spin chains is described as

$$
H=\frac{\lambda}{8 \pi^{2}} \sum_{k=1}^{L} H_{k k+1}+\xi \sum_{k=1}^{L} S_{1, k} \otimes S_{1, k+1} .
$$

For the undeformed part, $H_{k k+1}$ describes the nearest-neighbor interactions in the $s l(2)$ spin chain and the prefactor $\lambda / 8 \pi^{2}$ is now chosen for later convenience. For the deformation part, the $\xi$ is a constant parameter and the variable $S_{1, k}$ denotes the $s l(2)$ generator in the infinite-dimensional representation at the $k$-th site. For the detail of $S_{i}$, see Appendix A.

With the coherent state for the whole spin chain

$$
|\vec{n}\rangle \equiv \prod_{k=1}^{L}\left|\vec{n}_{k}\right\rangle
$$

the expectation value of $H$ is evaluated as

$$
\begin{aligned}
\langle\vec{n}|H| \vec{n}\rangle= & \frac{\lambda}{8 \pi^{2}} \sum_{k=1}^{L}\left\langle\vec{n}_{k} \vec{n}_{k+1}\left|H_{k k+1}\right| \vec{n}_{k} \vec{n}_{k+1}\right\rangle \\
& +\xi \sum_{k=1}^{L}\left\langle\vec{n}_{k}\left|S_{1, k}\right| \vec{n}_{k}\right\rangle\left\langle\vec{n}_{k+1}\left|S_{1, k+1}\right| \vec{n}_{k+1}\right\rangle .
\end{aligned}
$$

The undeformed part is computed in Appendix C. The deformation part becomes

$$
\left\langle\vec{n}_{k}\left|S_{1, k}\right| \vec{n}_{k}\right\rangle=\frac{1}{2} \sin \phi_{k} \sinh \rho_{k} .
$$

For the derivation, see Appendix B.

By taking the continuum limit, the expectation value of the deformed Hamiltonian with the coherent states is evaluated as

$$
\langle\vec{n}|H| \vec{n}\rangle \quad \longrightarrow \quad L \int d \sigma\left[\frac{\lambda}{32 \pi^{2} L^{2}}\left[\left(\partial_{\sigma} \rho\right)^{2}+\sinh ^{2} \rho\left(\partial_{\sigma} \phi\right)^{2}\right]+\frac{\xi}{4} \sin ^{2} \phi \sinh ^{2} \rho\right] .
$$

The deformation part contributes to the Hamiltonian as a non-derivative term. 
Then the Wess-Zumino term should be added to ensure the quantization condition at each site of the spin chain. Thus the total action is given by

$$
\begin{aligned}
S=\frac{L}{2} \int d t d \sigma\left[-C \sin ^{2} \phi \sinh ^{2} \rho+(\cosh \rho-1) \partial_{t} \phi\right. \\
\left.-\frac{\lambda}{16 \pi^{2} L^{2}}\left[\left(\partial_{\sigma} \rho\right)^{2}+\sinh ^{2} \rho\left(\partial_{\sigma} \phi\right)^{2}\right]\right],
\end{aligned}
$$

through the identification $\xi=2 C$. The action (3.20) agrees with the Landau-Lifshitz sigma models obtained from the string-theory side, up to the $\mathrm{S}^{1}$ circle.

\section{Time-like warped $\mathrm{AdS}_{3}$ and deformed spin chains}

\subsection{Fast-moving string limit}

We consider the string action on time-like warped $\mathrm{AdS}_{3} \times \mathrm{S}^{1}$ here. As in the case of spacelike warped $\mathrm{AdS}_{3}$, we first have to change the coordinate system of the metric (2.6). Again, we start from the metric (2.8) written with the group element and take the parametrization (3.1). Then the metric of the warped $\mathrm{AdS}_{3} \times \mathrm{S}^{1}$ is given by

$$
d s^{2}=R^{2}\left[-\cosh ^{2} \rho d t^{2}+d \rho^{2}+\sinh ^{2} \rho d \phi^{2}+d \varphi^{2}-C\left(\cosh ^{2} \rho d t+\sinh ^{2} \rho d \phi\right)^{2}\right],
$$

where an $\mathrm{S}^{1}$ circle is described by $\varphi$.

We perform here the same coordinate transformation as in (3.3) with the gauge $t=\kappa \tau$. Then the fast-moving limit (3.4) is taken for the same reason as mentioned in the previous. The resulting action of the warped $\mathrm{AdS}_{3} \times \mathrm{S}^{1}$ is given by

$$
\begin{aligned}
S=\frac{R^{2}}{4 \pi \alpha^{\prime}} \int d \tau d \sigma[ & -\kappa^{2} C \cosh ^{2} \tilde{\rho}+\kappa\left[(\cosh \tilde{\rho}-1) \partial_{\tau} \tilde{\phi}+2 \partial_{\tau} \tilde{\varphi}\right] \\
& \left.-\frac{1}{4}\left[\left(\partial_{\sigma} \tilde{\rho}\right)^{2}+2(\cosh \tilde{\rho}-1)\left(\partial_{\sigma} \tilde{\phi}\right)^{2}\right]-\left(\partial_{\sigma} \tilde{\phi}\right)^{2}\right] .
\end{aligned}
$$

The next task is to examine the Virasoro constraints under the limit (3.4). To the leading order in $\kappa$, the first Virasoro constraint (2.1) becomes

$$
0=\kappa\left[(\cosh \tilde{\rho}-1) \partial_{\sigma} \tilde{\phi}+2 \partial_{\sigma} \tilde{\varphi}\right] .
$$

By eliminating $\partial_{\sigma} \tilde{\varphi}$ from (4.2) with (4.3), the leading-order action is given by

$$
\begin{gathered}
S=\frac{R^{2}}{4 \pi \alpha^{\prime}} \int d \tau d \sigma\left[-\kappa^{2} C \cosh ^{2} \tilde{\rho}+\kappa\left[(\cosh \tilde{\rho}-1) \partial_{\tau} \tilde{\phi}+2 \partial_{\tau} \tilde{\varphi}\right]\right. \\
\left.-\frac{1}{4}\left[\left(\partial_{\sigma} \tilde{\rho}\right)^{2}+\sinh ^{2} \tilde{\rho}\left(\partial_{\sigma} \tilde{\phi}\right)^{2}\right]\right] .
\end{gathered}
$$

Note that the action (4.4) is written in the non-relativistic form again.

By introducing the following quantities,

$$
L \equiv \frac{R^{2} \kappa}{2 \pi \alpha^{\prime}}, \quad \lambda \equiv \frac{R^{4}}{\alpha^{\prime 2}},
$$


the action is rewritten as

$$
\begin{array}{r}
S=\frac{L}{2} \int d t d \sigma\left[-C \cosh ^{2} \tilde{\rho}+\left[(\cosh \tilde{\rho}-1) \partial_{t} \tilde{\phi}+2 \partial_{t} \tilde{\varphi}\right]\right. \\
\left.-\frac{\lambda}{16 \pi^{2} L^{2}}\left[\left(\partial_{\sigma} \tilde{\rho}\right)^{2}+\sinh ^{2} \tilde{\rho}\left(\partial_{\sigma} \tilde{\phi}\right)^{2}\right]\right] .
\end{array}
$$

where the time variable has been changed from $\tau$ to $t$ through $t=\kappa \tau$. As a result, a new potential term has been added. When $C=0$, the result in $[36,37]$ is reproduced again.

Finally let us see the equations of motion,

$$
\begin{array}{r}
\sinh \tilde{\rho} \partial_{t} \tilde{\rho}-\frac{\lambda}{8 \pi^{2} L^{2}} \partial_{\sigma}\left(\partial_{\sigma} \tilde{\phi} \sinh ^{2} \tilde{\rho}\right)=0, \\
-C \sinh \tilde{2 \rho}+\sinh \tilde{\rho} \partial_{t} \tilde{\phi}+\frac{\lambda}{8 \pi^{2} L^{2}}\left[\partial_{\sigma}^{2} \tilde{\rho}-\frac{1}{2} \sinh 2 \tilde{\rho}\left(\partial_{\sigma} \tilde{\phi}\right)^{2}\right]=0 .
\end{array}
$$

They are identical to the Landau-Lifshitz equations

$$
-\partial_{t} \vec{n}=\frac{\lambda}{8 \pi^{2} L^{2}} \vec{n} \times \partial_{\sigma}^{2} \vec{n}+\vec{n} \times \mathcal{J} \vec{n}
$$

with the anisotropic matrix $\mathcal{J}$

$$
\mathcal{J}^{i j}=\operatorname{diag}(j+2 C,-j,-j) \quad(j: \text { an arbitrary const. }) .
$$

Note that the new potential term in (4.6) is related to the following quantity,

$$
\mathcal{J}^{i j} n_{i} n_{j}=j+2 C \cosh ^{2} \tilde{\rho} .
$$

\subsection{From anisotropically deformed $\operatorname{sl}(2)$ spin chains}

Let us examine the continuum limit of the deformed $\operatorname{sl}(2)$ spin chains with coherent states.

The Hamiltonian of anisotropically deformed $s l(2)$ spin chains is described as

$$
H=\frac{\lambda}{8 \pi^{2}} \sum_{k=1}^{L} H_{k k+1}+\xi \sum_{k=1}^{L} S_{0, k} \otimes S_{0, k+1} .
$$

This is similar to the Hamiltonian (3.15). The only difference is that $S_{1, k}$ is replaced by $S_{0, k}$.

With the coherent state (3.16), the expectation value of $H$ is evaluated as

$$
\begin{aligned}
\langle\vec{n}|H| \vec{n}\rangle= & \frac{\lambda}{8 \pi^{2}} \sum_{k=1}^{L}\left\langle\vec{n}_{k} \vec{n}_{k+1}\left|H_{k k+1}\right| \vec{n}_{k} \vec{n}_{k+1}\right\rangle \\
& +\xi \sum_{k=1}^{L}\left\langle\vec{n}_{k}\left|S_{0, k}\right| \vec{n}_{k}\right\rangle\left\langle\vec{n}_{k+1}\left|S_{0, k+1}\right| \vec{n}_{k+1}\right\rangle .
\end{aligned}
$$

For the undeformed part, see Appendix C. The deformation part is computed like

$$
\left\langle\overrightarrow{n_{k}}\left|S_{0, k}\right| \overrightarrow{n_{k}}\right\rangle=\frac{1}{2} \cosh \rho_{k},
$$


as shown in Appendix B.

By taking the continuum limit, the expectation value is evaluated as

$$
\langle\vec{n}|H| \vec{n}\rangle \quad \longrightarrow \quad L \int d \sigma\left[\frac{\lambda}{32 \pi^{2} L^{2}}\left[\left(\partial_{\sigma} \rho\right)^{2}+\sinh ^{2} \rho\left(\partial_{\sigma} \phi\right)^{2}\right]+\frac{\xi}{4} \cosh ^{2} \rho\right] .
$$

The deformation part contributes to the Hamiltonian as a non-derivative term.

Then the Wess-Zumino term should be added to ensure the quantization condition at each site of the spin chain. Thus the total action is given by

$$
\begin{aligned}
S=\frac{L}{2} \int d t d \sigma[-C & \cosh ^{2} \rho+(\cosh \rho-1) \partial_{t} \phi \\
& \left.-\frac{\lambda}{16 \pi^{2} L^{2}}\left[\left(\partial_{\sigma} \rho\right)^{2}+\sinh ^{2} \rho\left(\partial_{\sigma} \phi\right)^{2}\right]\right],
\end{aligned}
$$

through the identification $\xi=2 C$. The action (4.16) agrees with the Landau-Lifshitz sigma models obtained in the string-theory side, up to the $\mathrm{S}^{1}$ circle.

\section{Null warped $\mathrm{AdS}_{3}$ and Jordanian deformations}

Finally we consider the case of strings propagating on null warped $\mathrm{AdS}_{3} \times \mathrm{S}^{1}$ here. We derive the Landau-Lifshitz sigma models from the string sigma models by taking the fast-moving limit. The resulting deformation term can also be correctly reproduced from Jordanian deformations of the $s l(2)$ spin chain by taking a continuum limit with coherent states.

\subsection{Fast-moving string limit}

For the case of null warped $\mathrm{AdS}_{3}$, there is a technical difficulty. It is because that the metric (2.9) is written with the Poincaré coordinates and we have to rewrite the metric (2.10) in terms of the global coordinate in the first place. The global coordinates for null warped $\mathrm{AdS}_{3}$ is discussed in [38], but it does not make sense to consider the fast-moving string limit.

A possible resolution is to start from the metric (2.10) with the group element and take the parametrization (3.1). Then the metric of null warped $\mathrm{AdS}_{3} \times \mathrm{S}^{1}$ is given by

$$
\begin{aligned}
& d s^{2}=R^{2}\left[d \rho^{2}-\cosh ^{2} \rho d t^{2}+\sinh ^{2} \rho d \phi^{2}+d \varphi^{2}\right. \\
& \quad-\frac{C}{2}[\cosh \rho(\cosh \rho+\sin (\phi-t) \sinh \rho) d t \\
& \left.\quad+\sinh \rho(\sinh \rho+\sin (\phi-t) \cosh \rho) d \phi-\cos (\phi-t) d \rho]^{2}\right],
\end{aligned}
$$

where an $\mathrm{S}^{1}$ circle is parametrized by $\varphi$. Note that there is no difficulty to consider the fast moving string limit.

Next we perform the same coordinate transformation as in (3.3) with the gauge $t=\kappa \tau$. Then the fast-moving limit (3.4) is taken for the same reason as mentioned in the previous. 
The resulting action on the warped $\mathrm{AdS}_{3} \times \mathrm{S}^{1}$ is given by

$$
\begin{aligned}
S=\frac{R^{2}}{4 \pi \alpha^{\prime}} \int d \tau d \sigma[ & -\kappa^{2} \frac{C}{2}(\cosh \tilde{\rho}+\sin \tilde{\phi} \sinh \tilde{\rho})^{2}+\kappa\left[(\cosh \tilde{\rho}-1) \partial_{\tau} \tilde{\phi}+2 \partial_{\tau} \tilde{\varphi}\right] \\
& \left.-\frac{1}{4}\left[\left(\partial_{\sigma} \tilde{\rho}\right)^{2}+2(\cosh \tilde{\rho}-1)\left(\partial_{\sigma} \tilde{\phi}\right)^{2}\right]-\left(\partial_{\sigma} \tilde{\varphi}\right)^{2}\right] .
\end{aligned}
$$

The Virasoro constraints are also changed under the limit (3.4). To the leading order in $\kappa$, the first Virasoro constraint (2.1) becomes

$$
0=\kappa\left[(\cosh \tilde{\rho}-1) \partial_{\sigma} \tilde{\phi}+2 \partial_{\sigma} \tilde{\varphi}\right] .
$$

which can be used to solve $\partial_{\sigma} \tilde{\varphi}$ in terms of $\partial_{\sigma} \tilde{\phi}$. After eliminating $\partial_{\sigma} \tilde{\varphi}$, to the leading-order action is given by

$$
\begin{gathered}
S=\frac{R^{2}}{4 \pi \alpha^{\prime}} \int d \tau d \sigma\left[-\frac{\kappa^{2} C}{2}(\cosh \tilde{\rho}+\sin \tilde{\phi} \sinh \tilde{\rho})^{2}+\kappa\left[(\cosh \tilde{\rho}-1) \partial_{\tau} \tilde{\phi}+2 \partial_{\tau} \tilde{\varphi}\right]\right. \\
\left.-\frac{1}{4}\left[\left(\partial_{\sigma} \tilde{\rho}\right)^{2}+\sinh ^{2} \tilde{\rho}\left(\partial_{\sigma} \tilde{\phi}\right)^{2}\right]\right] .
\end{gathered}
$$

Note that the action (5.4) is written in the non-relativistic form again.

By introducing new quantities like

$$
L \equiv \frac{R^{2} \kappa}{2 \pi \alpha^{\prime}}, \quad \lambda \equiv \frac{R^{4}}{\alpha^{\prime 2}},
$$

the following action is obtained,

$$
\begin{gathered}
S=\frac{L}{2} \int d t d \sigma\left[-\frac{C}{2}(\cosh \tilde{\rho}+\sin \tilde{\phi} \sinh \tilde{\rho})^{2}+\left[(\cosh \tilde{\rho}-1) \partial_{t} \tilde{\phi}+2 \partial_{t} \tilde{\varphi}\right]\right. \\
\left.-\frac{\lambda}{16 \pi^{2} L^{2}}\left[\left(\partial_{\sigma} \tilde{\rho}\right)^{2}+\sinh ^{2} \tilde{\rho}\left(\partial_{\sigma} \tilde{\phi}\right)^{2}\right]\right] .
\end{gathered}
$$

where the time variable has been changed from $\tau$ to $t$ through $t=\kappa \tau$. As a result, a new potential term has been added. When $C=0$, the result in $[36,37]$ is reproduced.

Finally let us see the equations of motion, which are derived from (5.6),

$$
\begin{aligned}
C \cos \tilde{\phi} \sinh \tilde{\rho}(\cosh \tilde{\rho}+\sin \tilde{\phi} \sinh \tilde{\rho})+\sinh \tilde{\rho} \partial_{t} \tilde{\rho}-\frac{\lambda}{8 \pi^{2} L^{2}} \partial_{\sigma}\left(\partial_{\sigma} \tilde{\phi} \sinh ^{2} \tilde{\rho}\right)=0, \\
-C\left[\sin \tilde{\phi} \cosh 2 \tilde{\rho}+\frac{1}{2}\left(1+\sin ^{2} \tilde{\phi}\right) \sinh 2 \tilde{\rho}\right]+\sinh \tilde{\rho} \partial_{t} \tilde{\phi} \\
+\frac{\lambda}{8 \pi^{2} L^{2}}\left[\partial_{\sigma}^{2} \tilde{\rho}-\frac{1}{2} \sinh 2 \tilde{\rho}\left(\partial_{\sigma} \tilde{\phi}\right)^{2}\right]=0 .
\end{aligned}
$$

These are identical to the Landau-Lifshitz equations

$$
-\partial_{t} \vec{n}=\frac{\lambda}{8 \pi^{2} L^{2}} \vec{n} \times \partial_{\sigma}^{2} \vec{n}+\vec{n} \times \mathcal{J} \vec{n},
$$


with the anisotropic matrix $\mathcal{J}$

$$
\mathcal{J}^{a b}=\left(\begin{array}{ccc}
j+C & C & 0 \\
C & -(j-C) & 0 \\
0 & 0 & -j
\end{array}\right) \quad(j \text { : an arbitrary const. }) .
$$

Note that the new potential term in (5.6) is related to the following quantity,

$$
\mathcal{J}^{i j} n_{i} n_{j}=j+C(\cosh \tilde{\rho}+\sin \tilde{\phi} \sinh \tilde{\rho})^{2} .
$$

\subsection{From Jordanian deformations of the $\operatorname{sl}(2)$ spin chain}

Let us take the continuum limit of Jordanian deformed $s l(2)$ spin chains with coherent states. The Jordanian deformed Hamiltonian is given by ${ }^{4}$

$$
H=\frac{\lambda}{8 \pi^{2}} \sum_{k=1}^{L} H_{k k+1}+\frac{\xi}{2} \sum_{k=1}^{L}\left(S_{0, k}+S_{1, k}\right) \otimes\left(S_{0, k+1}+S_{1, k+1}\right) .
$$

This is similar to the Hamiltonian (3.15) and (4.12). The only difference is that the variables in deformation part are replaced by $S_{0, k}+S_{1, k}$.

Then the expectation value of $H$ with the coherent state (3.16) is evaluated as

$$
\begin{aligned}
\langle\vec{n}|H| \vec{n}\rangle= & \frac{\lambda}{8 \pi^{2}} \sum_{k=1}^{L}\left\langle\vec{n}_{k} \vec{n}_{k+1}\left|H_{k k+1}\right| \vec{n}_{k} \vec{n}_{k+1}\right\rangle \\
& +\frac{\xi}{2} \sum_{k=1}^{L}\left\langle\vec{n}_{k}\left|\left(S_{0, k}+S_{1, k}\right)\right| \vec{n}_{k}\right\rangle\left\langle\vec{n}_{k+1}\left|\left(S_{0, k+1}+S_{1, k+1}\right)\right| \vec{n}_{k+1}\right\rangle .
\end{aligned}
$$

For the undeformed part, see Appendix C. The deformation part can be rewritten as

$$
\left\langle\vec{n}_{k}\left|\left(S_{0, k}+S_{1, k}\right)\right| \vec{n}_{k}\right\rangle=\frac{1}{2}\left(\cosh \rho_{k}+\sin \phi_{k} \sinh \rho_{k}\right),
$$

with the help of the formula in Appendix B.

By taking the continuum limit, the expectation value is given by

$$
\langle\vec{n}|H| \vec{n}\rangle \quad \longrightarrow \quad L \int d \sigma\left[\frac{\lambda}{32 \pi^{2} L^{2}}\left[\left(\partial_{\sigma} \rho\right)^{2}+\sinh ^{2} \rho\left(\partial_{\sigma} \phi\right)^{2}\right]+\frac{\xi}{8}(\cosh \rho+\sin \phi \sinh \rho)^{2}\right] .
$$

Note that the deformation part contributes to the Hamiltonian as a non-derivative term.

After adding the Wess-Zumino term to ensure the quantization condition at each site of the spin chain, the total action is given by

$$
\begin{array}{r}
S=\frac{L}{2} \int d t d \sigma\left[-\frac{C}{2}(\cosh \rho+\sin \phi \sinh \rho)^{2}+(\cosh \rho-1) \partial_{t} \phi\right. \\
\left.-\frac{\lambda}{16 \pi^{2} L^{2}}\left[\left(\partial_{\sigma} \rho\right)^{2}+\sinh ^{2} \rho\left(\partial_{\sigma} \phi\right)^{2}\right]\right]
\end{array}
$$

through the identification $\xi=2 C$. The action (5.12) agrees with the Landau-Lifshitz sigma models obtained from the string-theory side, up to the $\mathrm{S}^{1}$-contribution.

\footnotetext{
${ }^{4}$ Jordanian deformations of the XXX model are discussed in [39]. The Hamiltonian (5.10) is the $s l(2)$ analogue of the deformed Hamiltonian.
} 


\section{Conclusion and Discussion}

We have discussed the correspondence between string theories on warped $\mathrm{AdS}_{3}$ geometries in type IIB supergravity and anisotropic deformations of the $s l(2)$ spin chain. We have considered three kinds of warped $\mathrm{AdS}_{3}, 1$ ) space-like warped $\mathrm{AdS}_{3}, 2$ ) time-like warped $\mathrm{AdS}_{3}$ and 3) null warped $\mathrm{AdS}_{3}$. For the three examples, we have shown the agreement between the fast-moving limit of the relativistic string actions and the continuum limit of the corresponding deformed $s l(2)$ spin chains with $s l(2)$ coherent states.

Let us comment on the contribution of a constant three-form field strength $H_{3}=d B_{2}$, where $B_{2}$ is an NS-NS two-form field. The $H_{3}$ flux is contained in some supergravity solutions including the warped $\mathrm{AdS}_{3} \times \mathrm{S}^{1}$ geometries. Then we have to take account of it in our analysis because it induces a non-vanishing Wess-Zumino term ${ }^{5}$ in the string action and, as a result, the Landau-Lifshitz sigma models are also modified. So far, we have ignored it for simplicity. We will revisit this issue in another place.

It is also worth noting on a constant NS-NS $B$-field. In the string-theory side, it is basically irrelevant to our analysis because only closed strings are considered. Although it seems that a $B$-field, whose two indices are placed on warped $\mathrm{AdS}_{3}$ and $\mathrm{S}^{1}$ respectively, gives rise to a non-zero contribution, the $S^{1}$ part is mostly embedded into a larger space such as $\mathrm{S}^{3}$ and there is no contribution actually.

In the spin-chain side, the Wess-Zumino term should be added so as to rewrite the spin chain Hamiltonian into the Lagrangian form via the Legendre transformation, irrespective of $B_{2}$ and $H_{3}$ in the string-theory side. It can also be interpreted as the Berry phase. This interpretation enables us to see it as the contribution of a "fictitious" magnetic flux.

There are various open problems. First, it is of importance to take account of higherorder effects in the Landau-Lifshitz sigma models. Then it is necessary to consider a long-range generalization of Jordanian deformed spin chains like in the BDS spin chain [40]. The Bethe ansatz is also extended to the all-loop one probably by generalizing the work [41]. One should check the agreement between the classical energies of the spinning string solutions on warped AdS spaces and the energies of the corresponding Bethe string solutions.

We hope that our work would open a new arena for integrable gauge/string dualities.

\section{Acknowledgments}

We would like to thank I. Kawaguchi and T. Matsumoto for useful discussions. The work of $\mathrm{KY}$ was supported by the scientific grants from the Ministry of Education, Culture, Sports, Science and Technology (MEXT) of Japan (No. 22740160). This work was also supported in part by the Grant-in-Aid for the Global COE Program "The Next Generation of Physics, Spun from Universality and Emergence" from MEXT, Japan.

\footnotetext{
${ }^{5}$ This is different from the Wess-Zumino term in the spin-chain side. It exists without the $H_{3}$ flux.
} 


\section{A Convention of $\operatorname{sl}(2)$ generators}

The abstract Lie algebra $\operatorname{sl}(2)$ is formed by the generators $\hat{T}^{a}(a=0,1,2)$ satisfying the following commutation relations,

$$
\left[\hat{T}^{a}, \hat{T}^{b}\right]=\varepsilon^{a b}{ }_{c} \hat{T}^{c}
$$

Here we have introduced the totally antisymmetric tensor $\varepsilon^{a b c}$ with $\varepsilon^{012}=+1$ and the indices are lowered and raised with the $\gamma_{a b}=\operatorname{diag}(-1,+1,+1)$ and its inverse, respectively.

Then the light-cone notation is defined as

$$
\hat{T}^{ \pm} \equiv \frac{\hat{T}^{0} \pm \hat{T}^{1}}{\sqrt{2}} .
$$

It is useful to list the light-cone components of $\varepsilon^{a b}{ }_{c}$ and $\gamma_{a b}$ :

$$
\varepsilon^{-+}{ }_{2}=+1, \quad \gamma_{-+}=\gamma_{+-}=-1, \quad \gamma_{22}=+1 .
$$

\section{For the analysis of string sigma models}

In studying string sigma models on warped $\mathrm{AdS}_{3}$, we use the fundamental representation of the $s l(2)$ generators like

$$
D\left(\hat{T}^{0}\right) \equiv T^{0}=\frac{i}{2} \sigma_{2}, \quad D\left(\hat{T}^{1}\right) \equiv T^{1}=\frac{1}{2} \sigma_{1}, \quad D\left(\hat{T}^{2}\right) \equiv T^{2}=\frac{1}{2} \sigma_{3},
$$

where $\sigma_{i}(i=1,2,3)$ are the standard Pauli matrices. The generators are normalized as

$$
\operatorname{Tr}\left(T^{a} T^{b}\right)=\frac{1}{2} \gamma^{a b}
$$

It is helpful to introduce the left-invariant one-form $J$ defined as

$$
J \equiv g^{-1} d g .
$$

It is expanded in terms of the generators $T^{a}$ like

$$
\begin{aligned}
J & =-T^{0} J^{0}+T^{1} J^{1}+T^{2} J^{2} \\
& =-T^{+} J^{-}-T^{-} J^{+}+T^{2} J^{2},
\end{aligned}
$$

where $J^{a} \equiv 2 \operatorname{Tr}\left(T^{a} J\right)$.

\section{For the analysis of $\operatorname{sl}(2)$ spin chains}

In the analysis of $s l(2)$ spin chains, we use the infinite-dimensional representation of the $s l(2)$ generators by following the notation in [37],

$$
D^{(\infty)}\left(\hat{T}^{0}\right) \equiv i S_{0}, \quad D^{(\infty)}\left(\hat{T}^{1}\right) \equiv-i S_{1}, \quad D^{(\infty)}\left(\hat{T}^{2}\right) \equiv i S_{2} .
$$

Here the generators $S_{i}$ are operators acting on a Hilbert space (rather than matrices) and satisfy the commutation relations,

$$
\left[S_{0}, S_{1}\right]=i S_{2}, \quad\left[S_{1}, S_{2}\right]=-i S_{0}, \quad\left[S_{2}, S_{0}\right]=i S_{1}
$$


We introduce the operators $S_{ \pm}$defined as

$$
S_{ \pm} \equiv S_{2} \mp i S_{1}
$$

Then the commutation relations are rewritten into the following form,

$$
\left[S_{-}, S_{+}\right]=2 S_{0}, \quad\left[S_{0}, S_{ \pm}\right]= \pm S_{ \pm}
$$

The light-cone components $\hat{T}^{ \pm}$are represented by

$$
D^{(\infty)}\left(\hat{T}^{ \pm}\right)=\frac{i}{\sqrt{2}}\left(S_{0} \mp S_{1}\right) .
$$

\section{B The $\operatorname{sl}(2)$ coherent states}

In taking the continuum limit of spin chains, one has to use the $\operatorname{sl}(2)$ coherent states [42]. We follow the notation in [37].

To define the $s l(2)$ coherent states, let us introduce a unit vector $\vec{n}$ described by

$$
\vec{n}=\left(n_{i}\right)=(\cosh \rho, \sinh \rho \sin \phi, \sinh \rho \cos \phi) .
$$

The inner product of $\vec{n}$ is defined as

$$
\vec{n}^{2} \equiv \eta^{i j} n_{i} n_{j}=n_{0}^{2}-n_{1}^{2}-n_{2}^{2}=1 \quad \text { (hyperboloid) }
$$

and the indices are raised and lowered with $\eta^{i j}=\operatorname{diag}(+1,-1,-1)$.

By using the unit vector, the $\operatorname{sl}(2)$ coherent states are defined as [37]

$$
|\vec{n}\rangle \equiv \mathrm{e}^{\xi S_{+}-\bar{\xi} S_{-}}|0\rangle, \quad \xi=\frac{1}{2} \rho \mathrm{e}^{i \phi},
$$

where $|0\rangle$ is the lowest-weight state with spin $1 / 2$ and satisfies

$$
S_{0}|0\rangle=\frac{1}{2}|0\rangle, \quad S_{-}|0\rangle=0 .
$$

The expectation value of $S_{i}$ with the coherent states is evaluated as

$$
\left\langle\vec{n}\left|S_{i}\right| \vec{n}\right\rangle=\frac{1}{2} n_{i}
$$

and this relation is useful to take the continuum limit of the spin chains.

\section{The continuum limit of the $\operatorname{sl}(2)$ spin chain}

To be self-contained, we shall give a short review on the continuum limit of the $s l(2)$ spin chain by sandwiching the spin-chain Hamiltonian in the $s l(2)$ coherent states introduced in Appendix B. 
In the abstract form, the Hamiltonian of the $s l(2)$ spin chain with the nearest-neighbor interactions is given by

$$
H=\frac{\lambda}{8 \pi^{2}} \sum_{k=1}^{L} H_{k k+1},
$$

where $L$ is the length of chain and the coefficients are chosen for later convenience ${ }^{6}$. For the detail of the Hamiltonian, see the references [36, 37, 43]. This spin chain is often called the $\mathrm{XXX}_{-1 / 2}$ Heisenberg spin chain.

The coherent state for the whole spin chain is given by

$$
|\vec{n}\rangle \equiv \prod_{k=1}^{L}\left|\vec{n}_{k}\right\rangle
$$

and the expectation value of $H$ is computed as

$$
\langle\vec{n}|H| \vec{n}\rangle=\frac{\lambda}{8 \pi^{2}} \sum_{k=1}^{L} \log \left(1-\frac{\left(\vec{n}_{k}-\vec{n}_{k+1}\right)^{2}}{4}\right) .
$$

To take a continuum limit, the vector at each site should be replaced as

$$
\vec{n}_{k} \quad \rightarrow \quad \vec{n}(\sigma)=\vec{n}\left(\frac{k}{L}\right)
$$

and the difference is expanded in $L \rightarrow \infty$ limit like

$$
\vec{n}_{k+1}-\vec{n}_{k}=\frac{1}{L} \partial_{\sigma} \vec{n}+\mathcal{O}\left(\frac{1}{L^{2}}\right)
$$

In the limit $L \rightarrow \infty$ with $\lambda / L^{2}=$ fixed, the expectation value (C.2) is rewritten as

$$
\langle\vec{n}|H| \vec{n}\rangle \quad \longrightarrow \quad \frac{\lambda}{8 \pi^{2}} L \int d \sigma \frac{1}{4 L^{2}}\left[\left(\partial_{\sigma} \rho\right)^{2}+\sinh ^{2} \rho\left(\partial_{\sigma} \phi\right)^{2}\right] .
$$

To ensure the quantization condition at each site of the spin chain, it is necessary to add the Wess-Zumino term [35],

$$
S_{\mathrm{WZ}}=-\frac{1}{2} \sum_{k=1}^{L} \int d t \int_{0}^{1} d z \vec{n}_{k} \cdot\left(\partial_{z} \vec{n}_{k} \times \partial_{t} \vec{n}_{k}\right)=\frac{1}{2} \int d t \sum_{k=1}^{L}\left(\cosh \rho_{k}-1\right) \partial_{t} \phi_{k} .
$$

Thus the continuum limit of the (undeformed) $s l(2)$ spin chain leads to the total action,

$$
S=\frac{L}{2} \int d t d \sigma\left[(\cosh \rho-1) \partial_{t} \phi-\frac{\lambda}{16 \pi^{2} L^{2}}\left[\left(\partial_{\sigma} \rho\right)^{2}+\sinh ^{2} \rho\left(\partial_{\sigma} \phi\right)^{2}\right]\right]
$$

\footnotetext{
${ }^{6}$ In the usual $\mathrm{AdS}_{5} / \mathrm{CFT}_{4}$, the prefactor can be computed by perturbative computation in $\mathcal{N}=4 \mathrm{SYM}$. However, it is not possible in the present case because the field-theory action has not been clarified yet. At most, it can be fixed so as to agree with the string-theory result.
} 


\section{References}

[1] J. M. Maldacena, "The large N limit of superconformal field theories and supergravity," Adv. Theor. Math. Phys. 2 (1998) 231 [Int. J. Theor. Phys. 38 (1999) 1113]. [arXiv:hep-th/9711200].

[2] S. S. Gubser, I. R. Klebanov and A. M. Polyakov, "Gauge theory correlators from non-critical string theory," Phys. Lett. B 428 (1998) 105 [arXiv:hep-th/9802109].

[3] E. Witten, "Anti-de Sitter space and holography," Adv. Theor. Math. Phys. 2 (1998) 253 [arXiv:hep-th/9802150].

[4] N. Beisert et al., "Review of AdS/CFT Integrability: An Overview," arXiv:1012.3982 [hep-th].

[5] N. Beisert and P. Koroteev, "Quantum Deformations of the One-Dimensional Hubbard Model," J. Phys. A 41 (2008) 255204 [arXiv:0802.0777 [hep-th]].

[6] N. Beisert, W. Galleas and T. Matsumoto, "A Quantum Affine Algebra for the Deformed Hubbard Chain,” J. Phys. A 45 (2012) 365206 [arXiv:1102.5700 [math-ph]].

[7] B. Hoare, T. J. Hollowood and J. L. Miramontes, " $q$-Deformation of the $\mathrm{AdS}_{5} \times \mathrm{S}^{5}$ Superstring S-matrix and its Relativistic Limit," JHEP 1203 (2012) 015 [arXiv:1112.4485 [hep-th]]; "Bound States of the $q$-Deformed $\mathrm{AdS}_{5} \times \mathrm{S}^{5}$ Superstring S-matrix," JHEP 1210 (2012) 076 [arXiv:1206.0010 [hep-th]]; "Restoring Unitarity in the $q$-Deformed World-Sheet S-Matrix," arXiv:1303.1447 [hep-th].

[8] M. de Leeuw, V. Regelskis and A. Torrielli, "The Quantum Affine Origin of the AdS/CFT Secret Symmetry," J. Phys. A 45 (2012) 175202 [arXiv:1112.4989 [hep-th]].

[9] G. Arutyunov, M. de Leeuw and S. J. van Tongeren, "The Quantum Deformed Mirror TBA I," JHEP 1210 (2012) 090 [arXiv:1208.3478 [hep-th]]; "The Quantum Deformed Mirror TBA II," JHEP [JHEP 1302 (2013) 012] [arXiv:1210.8185 [hep-th]].

[10] I. V. Cherednik, "Relativistically Invariant Quasiclassical Limits Of Integrable Two-Dimensional Quantum Models," Theor. Math. Phys. 47 (1981) 422 [Teor. Mat. Fiz. 47 (1981) 225].

[11] L. D. Faddeev and N. Y. Reshetikhin, "Integrability of the principal chiral field model in (1+1)-dimension," Annals Phys. 167 (1986) 227.

[12] J. Balog, P. Forgacs and L. Palla, "A Two-dimensional integrable axionic sigma model and T duality," Phys. Lett. B 484 (2000) 367 [hep-th/0004180].

[13] W. -Y. Wen, "Spin chain from marginally deformed $\mathrm{AdS}_{3} \times \mathrm{S}^{3}$," Phys. Rev. D 75 (2007) 067901 [hep-th/0610147].

[14] I. Kawaguchi and K. Yoshida, "Hidden Yangian symmetry in sigma model on squashed sphere," JHEP 1011 (2010) 032. [arXiv:1008.0776 [hep-th]].

[15] D. Orlando, S. Reffert and L. I. Uruchurtu, "Classical integrability of the squashed three-sphere, warped $\mathrm{AdS}_{3}$ and Schrödinger spacetime via T-Duality," J. Phys. A 44 (2011) 115401. [arXiv:1011.1771 [hep-th]].

[16] I. Kawaguchi, D. Orlando and K. Yoshida, "Yangian symmetry in deformed WZNW models on squashed spheres," Phys. Lett. B 701 (2011) 475. [arXiv:1104.0738 [hep-th]].

[17] I. Kawaguchi and K. Yoshida, "Hybrid classical integrability in squashed sigma models," Phys. Lett. B 705 (2011) 251 [arXiv:1107.3662 [hep-th]]; "Hybrid classical integrable 
structure of squashed sigma models: A short summary,” J. Phys. Conf. Ser. 343 (2012) 012055 [arXiv:1110.6748 [hep-th]].

[18] I. Kawaguchi and K. Yoshida, "Classical integrability of Schrödinger sigma models and q-deformed Poincare symmetry," JHEP 1111 (2011) 094 [arXiv:1109.0872 [hep-th]]; "Exotic symmetry and monodromy equivalence in Schrödinger sigma models," JHEP 1302 (2013) 024 [arXiv:1209.4147 [hep-th]].

[19] I. Kawaguchi, T. Matsumoto and K. Yoshida, "The classical origin of quantum affine algebra in squashed sigma models," JHEP 1204 (2012) 115 [arXiv:1201.3058 [hep-th]];

"On the classical equivalence of monodromy matrices in squashed sigma model," JHEP 1206 (2012) 082 [arXiv:1203.3400 [hep-th]].

[20] B. Basso and A. Rej, "On the integrability of two-dimensional models with $U(1) \times S U(N)$ symmetry," Nucl. Phys. B 866 (2013) 337 [arXiv:1207.0413 [hep-th]].

[21] M. J. Duff, H. Lu and C. N. Pope, " $\mathrm{AdS}_{3} \times \mathrm{S}^{3}$ (un)twisted and squashed, and an $O(2,2, \mathbb{Z})$ multiplet of dyonic strings," Nucl. Phys. B 544 (1999) 145 [hep-th/9807173].

[22] M. Rooman and P. Spindel, "Godel metric as a squashed anti-de Sitter geometry," Class. Quant. Grav. 15 (1998) 3241 [gr-qc/9804027].

[23] D. Israel, "Quantization of heterotic strings in a Godel / anti-de Sitter space-time and chronology protection," JHEP 0401 (2004) 042 [hep-th/0310158].

[24] S. Detournay, D. Orlando, P. M. Petropoulos and P. Spindel, "Three-dimensional black holes from deformed anti-de Sitter," JHEP 0507 (2005) 072 [hep-th/0504231].

[25] D. T. Son, "Toward an AdS/cold atoms correspondence: A Geometric realization of the Schrodinger symmetry," Phys. Rev. D 78 (2008) 046003 [arXiv:0804.3972 [hep-th]].

[26] K. Balasubramanian and J. McGreevy, "Gravity duals for non-relativistic CFTs," Phys. Rev. Lett. 101 (2008) 061601 [arXiv:0804.4053 [hep-th]].

[27] D. Anninos, W. Li, M. Padi, W. Song and A. Strominger, "Warped $\mathrm{AdS}_{3}$ Black Holes," JHEP 0903 (2009) 130 [arXiv:0807.3040 [hep-th]].

[28] G. Compere, S. Detournay and M. Romo, "Supersymmetric Godel and warped black holes in string theory," Phys. Rev. D 78 (2008) 104030 [arXiv:0808.1912 [hep-th]].

[29] D. Anninos, "Hopfing and Puffing Warped Anti-de Sitter Space," JHEP 0909 (2009) 075 [arXiv:0809.2433 [hep-th]].

[30] T. S. Levi, J. Raeymaekers, D. Van den Bleeken, W. Van Herck and B. Vercnocke, "Godel space from wrapped M2-branes," JHEP 1001 (2010) 082 [arXiv:0909.4081 [hep-th]].

[31] D. Orlando and L. I. Uruchurtu, "Warped anti-de Sitter spaces from brane intersections in type II string theory," JHEP 1006 (2010) 049 [arXiv:1003.0712 [hep-th]].

[32] E. O. Colgain and H. Samtleben, "3D gauged supergravity from wrapped M5-branes with AdS/CMT applications," JHEP 1102 (2011) 031 [arXiv:1012.2145 [hep-th]].

[33] N. Bobev and B. C. van Rees, "Schrodinger Deformations of $A d S_{3} x S^{3}$," JHEP 1108 (2011) 062 [arXiv:1102.2877 [hep-th]].

[34] W. Song and A. Strominger, "Warped $\mathrm{AdS}_{3} /$ Dipole-CFT Duality," arXiv:1109.0544 [hep-th].

[35] M. Kruczenski, "Spin chains and string theory," Phys. Rev. Lett. 93 (2004) 161602 [hep-th/0311203]. 
[36] B. Stefanski, Jr. and A. A. Tseytlin, "Large spin limits of AdS/CFT and generalized Landau-Lifshitz equations," JHEP 0405 (2004) 042 [hep-th/0404133].

[37] S. Bellucci, P. -Y. Casteill, J. F. Morales and C. Sochichiu, "SL(2) spin chain and spinning strings on $\mathrm{AdS}_{5} \times \mathrm{S}^{5}$," Nucl. Phys. B 707 (2005) 303 [hep-th/0409086].

[38] M. Blau, J. Hartong and B. Rollier, "Geometry of Schrodinger Space-Times, Global Coordinates, and Harmonic Trapping," JHEP 0907 (2009) 027 [arXiv:0904.3304 [hep-th]].

[39] A. Stolin and P. P. Kulish, "New rational solutions of Yang-Baxter equation and deformed Yangians," Czech. J. Phys. 47 (1997) 123 [arXiv:q-alg/9608011].

[40] N. Beisert, V. Dippel, andM. Staudacher, "A Novel long range spin chain and planar $\mathrm{N}=4$ super Yang-Mills," JHEP 0407 (2004) 075 [hep-th/0405001].

[41] V. A. Kazakov and K. Zarembo, "Classical / quantum integrability in non-compact sector of AdS/CFT," JHEP 0410 (2004) 060 [hep-th/0410105].

[42] A. Perelomov, "Generalized Coherent States and their Applications," Springer-Verlag, Berlin, 1986.

[43] N. Beisert, "The complete one loop dilatation operator of $\mathrm{N}=4$ superYang-Mills theory," Nucl. Phys. B 676 (2004) 3 [hep-th/0307015]; N. Beisert and M. Staudacher, "The N=4 SYM integrable super spin chain," Nucl. Phys. B 670 (2003) 439 [hep-th/0307042]. 